\title{
Spectral tunability of laser-induced spin dynamics in the ferromagnetic semiconductor $\mathrm{CdCr}_{2} \mathrm{Se}_{4}$
}

\author{
A. Pogrebna,${ }^{1, *}{ }^{* \dagger}$ S. Barsaume,${ }^{1, \dagger}$ R. R. Subkhangulov,${ }^{1}$ A. V. Telegin,,${ }^{2}$ Yu. P. Sukhorukov, ${ }^{2}$ \\ A. V. Chzhan, ${ }^{3}$ Th. Rasing, ${ }^{1}$ and A. V. Kimel ${ }^{1,4, *}$ \\ ${ }^{1}$ Radboud University, Institute for Molecules and Materials, 6525 ED Nijmegen, The Netherlands \\ ${ }^{2}$ Miheev Institute of Metal Physics Ural Branch of Russian Academy of Sciences, 620990 Yekaterinburg, Russia \\ ${ }^{3}$ Siberian Federal University, 660041 Krasnoyarsk, Russia \\ ${ }^{4}$ Russian Technological University (MIREA), Moscow 119454, Russia
}

(Received 13 June 2018; revised manuscript received 12 September 2018; published 14 December 2018)

\begin{abstract}
Here we report that femtosecond laser pulses are able to trigger oscillations of the magneto-optical Faraday rotation in the ferromagnetic semiconductor $\mathrm{CdCr}_{2} \mathrm{Se}_{4}$ in the presence of an applied magnetic field. The frequency of these oscillations is a linear function of the magnetic field and corresponds to the ferromagnetic resonance (FMR). Tuning the photon energy of the pump pulses we reveal two different mechanisms, which induce FMR precession in this material. In the case of pumping from the valence band deep into the conduction band (photon energy $3.1 \mathrm{eV}$ ), the phase of the spin oscillations is not sensitive to the polarization of the pump, but can be reversed over $180 \mathrm{deg}$ by changing the polarity of the applied magnetic field. We assign these oscillations to the coherent spin precession triggered by ultrafast laser-induced heating. This mechanism requires a strong optical absorption in the material and becomes inactive if the pump photon energy is below the band gap. Tuning the photon energy in a wide range from 0.88 to $2.1 \mathrm{eV}$ reveals the second mechanism of optical excitation of coherent spin oscillations with a maximum around $1.2 \mathrm{eV}$, i.e., very close to the energy of the band gap in the semiconductor. Contrary to the laser-induced heating, this excitation mechanism is pump polarization dependent, being the most efficient if the pump is circularly polarized. The phase of the spin oscillations is independent of the polarity of the applied magnetic field, but changes by 180 deg under reversing the helicity of light. We suggest that the effect can be interpreted in terms of spin transfer torque experienced by the network of the ordered $\mathrm{Cr}^{3+}$ spins as a result of excitation of electrons from the top of the $p$-type valence band to the bottom of the $s$-type conduction band. In particular, a strong spin-orbit interaction experienced by the carriers in the valence band is responsible for the coupling of the spins of the photogenerated carriers and the polarization of light. Due to strong $p d$ - and $s d$-exchange interactions the spins of the photocarriers appear to be coupled to the network of ordered spins of the $\mathrm{Cr}^{3+}$ ions.
\end{abstract}

DOI: 10.1103/PhysRevB.98.214427

\section{INTRODUCTION}

The idea of changing the magnetic properties of media with the help of light has been intriguing people for a long time $[1,2]$. This became especially appealing after the development of solid-state femtosecond laser sources which are able to generate sub-100-fs laser pulses. These developments naturally raised questions about the mechanisms of laser-induced magnetization dynamics and the speed limit of the optical control of magnetism [3-7]. It has been demonstrated that magnetization dynamics can be triggered via several distinct mechanisms. In 1996 the work of Beaurepaire et al. [1] started the field of ultrafast magnetization dynamics by reporting laser-induced demagnetization in $\mathrm{Ni}$ thin films, using

\footnotetext{
*a.pogrebna@science.ru.nl, a.kimel@science.ru.nl

${ }^{\dagger}$ A. Pogrebna and S. Barsaume contributed equally to this work.
}

Published by the American Physical Society under the terms of the Creative Commons Attribution 4.0 International license. Further distribution of this work must maintain attribution to the author(s) and the published article's title, journal citation, and DOI.
60 -fs pulses. The mechanism was discussed in terms of a three-temperature model, in which light first interacts with free electrons, rapidly increasing the electronic temperature. Afterwards the excitation is transferred via the lattice to the spins. Demonstration of the inverse Faraday effect in dielectric iron oxides $\left(\mathrm{DyFeO}_{3}\right.$ and yttrium iron garnet) led to another mechanism of laser-induced spin precession in magnetic materials [2,3]. Contrary to the previous one, this mechanism did not rely on heating but required circularly polarized laser excitation. The phase of the spin oscillations was independent of the direction of the applied magnetic field, but a change of the pump helicity resulted in a 180-deg change of the phase of the oscillations.

Soon after the first reports of the helicity dependent laser excitation in magnetic dielectrics, pump polarization dependent excitations and even magnetization reversal were also reported for metallic GdFeCo [8]. The majority of the experimental studies in this field concern either insulating or metallic media [9]. Investigation of laser-induced spin dynamics in semiconductors has achieved far less attention, being limited to very few compounds [9-17]. It is remarkable that practically in all these magnetic semiconductors it was possible to excite spin precession via a helicity independent 
mechanism based on a relatively simple laser-induced heating $[11,12]$ or even more complex scenario $[10,18]$, while helicity dependent laser-induced spin dynamics was reported only for GaMnAs and EuO [15,19]. Aiming to understand the role of the polarization and photon energy in defining the dominant mechanism of the laser-induced spin dynamics in semiconductors, we performed spectral studies of the laser-induced spin dynamics in the ferromagnetic thin-film semiconductor $\mathrm{CdCr}_{2} \mathrm{Se}_{4}$. This material has a relatively high Curie temperature $T_{\mathrm{C}}=130 \mathrm{~K}$ and a band gap around $1.2 \mathrm{eV}$ at $4 \mathrm{~K}$ [20-23]. Only helicity independent spin precession excited with photons with energy of $3.1 \mathrm{eV}$ has been reported for this semiconductor so far [24]. At the same time, it is known that this compound possesses strong magneto-optical and photomagnetic effects [25-27]. All these features make $\mathrm{CdCr}_{2} \mathrm{Se}_{4}$ an attractive candidate for such a spectral and polarization dependent study of the mechanisms of interaction of light with spins.

In this paper we present the observation of ferromagnetic precession triggered by sub-100-fs laser pulses in two different absorption regimes. In the first part we show that pump pulses with a photon energy much higher than the band gap can induce magnetization precession via a polarization independent heating mechanism. Contrary, if the photon energy is close to the energy of the semiconductor band gap, the ferromagnetic precession is excited by means of a polarization dependent mechanism of optical spin transfer torque.

\section{SAMPLES AND EXPERIMENTAL TECHNIQUE}

$\mathrm{CdCr}_{2} \mathrm{Se}_{4}$ investigated here is a single-sublattice ferromagnetic semiconductor with the Curie temperature $T_{\mathrm{C}}=130 \mathrm{~K}$ [28]. Strong ferromagnetic superexchange between nearestneighbor chromium $\mathrm{Cr}^{3+}$ ions through $\mathrm{Cd}$ is the main type of interaction responsible for the ferromagnetic order in this material. $\mathrm{CdCr}_{2} \mathrm{Se}_{4}$ has a band gap around $1.2 \mathrm{eV}$ at $4 \mathrm{~K}$ and $1.4 \mathrm{eV}$ at $78 \mathrm{~K}[20,21]$.

The polycrystalline film of thickness $d=100 \mathrm{~nm}$ was obtained by vacuum deposition [29] on a borosilicate glass substrate of thickness $d=0.14 \mathrm{~mm}$ at $T_{\text {substr }}=250^{\circ} \mathrm{C}$. The initial components $\mathrm{Cd}, \mathrm{Cr}$, and Se were placed in three melting pots with independent adjustment of the evaporation rate of each element described. The as-grown amorphous film was annealed in vacuum at $T=450^{\circ} \mathrm{C}$ for $2 \mathrm{~h}$. The composition of the film was checked by x-ray diffraction with an accuracy of 5\%. Such a polycrystalline film has an easy-plane type of anisotropy and does not show any measurable anisotropy in the sample plane.

To study ultrafast laser-induced spin dynamics, a standard optical pump-probe technique was applied, with 50-fs optical pulses from a 1-kHz Ti: $\mathrm{Al}_{2} \mathrm{O}_{3}$ regenerative amplifier seeded with a Ti: $\mathrm{Al}_{2} \mathrm{O}_{3}$ oscillator. Two optical pulses, suitably delayed in time with respect to each other, arrive at the sample [see inset Fig. 1(a)]. One stronger, circularly polarized, beam (pump) drives the sample out of equilibrium, while another weaker, linearly polarized, beam (probe) is used to detect the pump-induced changes with a time resolution of a pulse duration. In all the experiments, for the probe we used photons with an energy of $1.55 \mathrm{eV}$, which is above the band gap. The

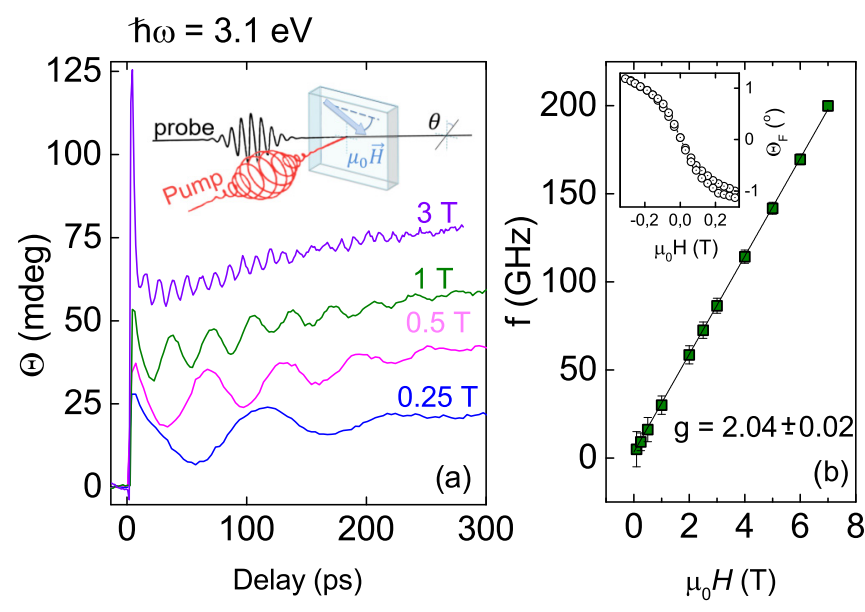

FIG. 1. (a) Transient magneto-optical Faraday rotation induced by pumping with a photon energy of $3.1 \mathrm{eV}$ at different magnetic fields. The measurements were performed at $10 \mathrm{~K}$. The external magnetic field is applied at an angle $\sim 20 \mathrm{deg}$ from the surface of the sample. (b) The frequency of the oscillations observed in panel (a) as a function of the applied magnetic field. The frequencies were deduced with the help of FFT analysis. The inset in panel (a) shows the schematic of the experiment. The inset in panel (b) shows the static magneto-optical Faraday rotation, measured with an out-of-plane magnetic field, by means of a cw laser at the wavelength of $800 \mathrm{~nm}$ at $78 \mathrm{~K}$.

pump photon energy was tuned from 0.88 to $3.1 \mathrm{eV}$. The circularly polarized pump pulses were incident under a small angle with respect to the sample normal. The probe pulses were at normal incidence to the sample surface. The pump beam was focused on the sample to a spot size of $100 \mu \mathrm{m}$, and the probe spot size was around $50 \mu \mathrm{m}$. The energy of the pump pulse was around $1 \mu \mathrm{J}$ and the probe pulse was about 30 times weaker. After transmission through the sample, the polarization rotation of the probe pulses was detected by a balanced detector as a function of time delay between pump and probe pulses. To activate both pump polarization dependent and pump polarization independent mechanisms, i.e., heat-induced mechanisms of spin precession, a magnetic field up to $7 \mathrm{~T}$ was applied at an angle of about $20 \mathrm{deg}$ with respect to the plane of the sample [see inset Fig. 1(a)]. To suppress the polarization independent mechanism, but keep the polarization dependent one unaffected, the field was applied in the sample plane. The temperature of the sample was varied with the help of a cryostat.

The rotation of the probe polarization, $\theta$, measured in this way corresponds to the magneto-optical Faraday effect, which is proportional to the projection of the magnetization on the wave vector of the probe light, i.e., the magnetization projection on the sample normal. If light triggers homogeneous spin precession, the magnetization component normal to the sample plane, and thus the Faraday rotation, should oscillate at the frequency of ferromagnetic resonance. We exclude the contribution of magnetooptical effects of higher orders (e.g., magnetic linear dichroism) [30]. In particular, we have checked and confirmed the independence of the observed dynamics on the polarization of the incidence probe pulse. 

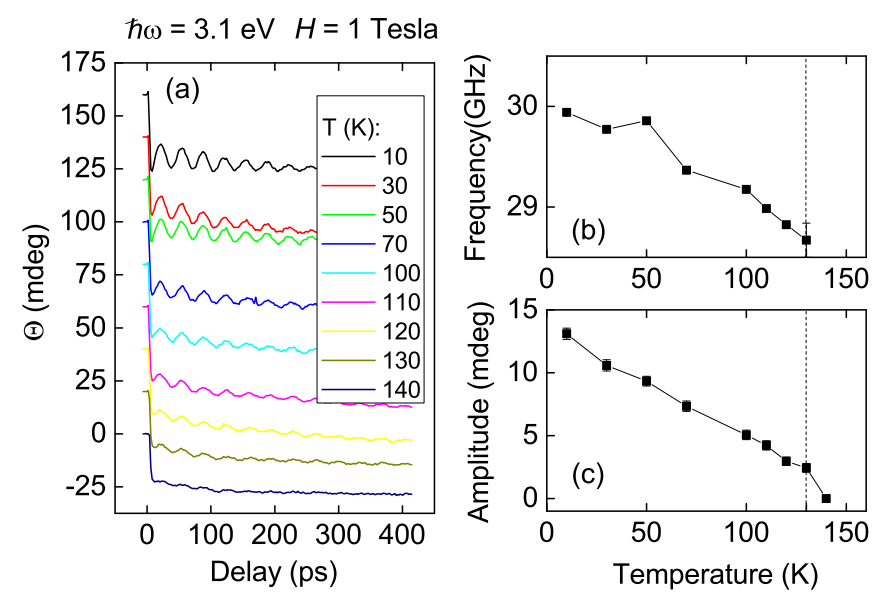

FIG. 2. (a) Transient magneto-optical Faraday effect induced by pump pulses with a photon energy of $3.1 \mathrm{eV}$ measured at different temperatures at $1 \mathrm{~T}$. The external magnetic field was applied at an angle $\sim 20$ deg from the sample surface. Both (b) and (c) show the decrease of the frequency and the amplitude of the oscillations while crossing the Curie temperature of $130 \mathrm{~K}$.

\section{RESULTS}

\section{A. Pump photon energy $\hbar \omega=3.1 \mathrm{eV}$ : Excitation above the gap}

To reveal the mechanism of the excitation of laser-induced spin dynamics in a strong absorption regime, we employed circularly polarized pump pulses with a photon energy of $3.1 \mathrm{eV}$. This energy is much higher than the band gap of $\mathrm{CdCr}_{2} \mathrm{Se}_{4}$. In a small magnetic field of $0.25 \mathrm{~T}$, applied at an angle of about $20 \mathrm{deg}$ with respect to the sample surface, at a temperature of $10 \mathrm{~K}$, we observed damped oscillations of the transient polarization rotation with a frequency of $10 \mathrm{GHz}$ [see Fig. 1(a)]. The frequency of these oscillations increased upon an increase of the magnetic field. Figure 1(a) shows the dynamics of the transient polarization rotation. A fast Fourier-transform (FFT) analysis shows that the frequency of the oscillations is a linear function of the applied magnetic field, with a slope corresponding to the Landé $g$ factor of 2 [see Fig. 1(b)].

The dynamics of the polarization rotation measured at various temperatures is shown in Fig. 2. The frequency of the oscillations weakly depends on temperature, and their amplitude [see Figs. 2(b) and 2(c)] drops to zero just above $130 \mathrm{~K}$. This temperature corresponds to the Curie point.

Hence, the observed dynamics, measured at different magnetic fields and temperatures, suggests that the observed oscillations correspond to a coherent spin precession of $\mathrm{Cr}^{3+}$ ions. Interestingly, a comparison of the values of the static magnetooptical Faraday effect [see inset Fig. 1(b)] with the amplitude of the laser-induced oscillation allows us to estimate the angle of deviation of the coherently precessing magnetization from its equilibrium position. Regarding the fact that the total Faraday rotation measured at the wavelength of $800 \mathrm{~nm}$ at $78 \mathrm{~K}$ is about $1.8 \mathrm{deg}$, one can roughly estimate the angle of precession to be around $0.5 \mathrm{deg}$.

In an attempt to reveal the mechanism responsible for the excitation of the spins, we performed the measurements for two helicities of the pump pulses $\left(\sigma^{+}\right.$and $\left.\sigma^{-}\right)$and two

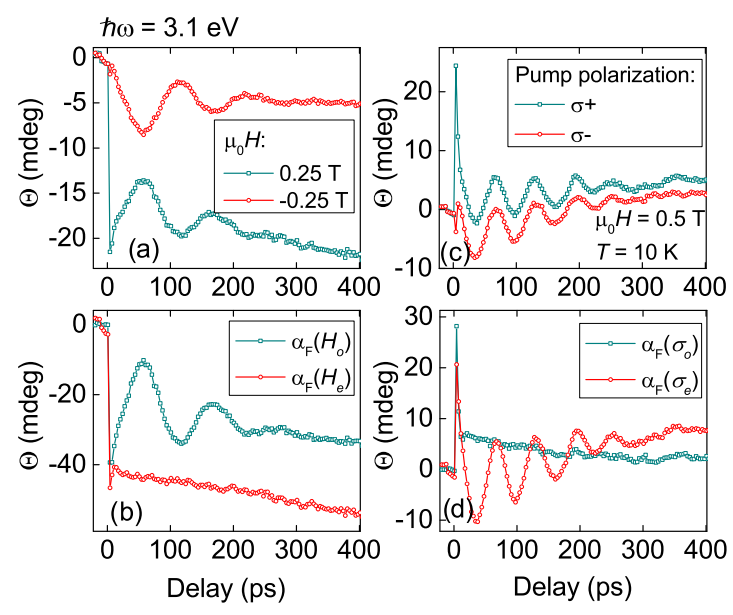

FIG. 3. (a) Transient magneto-optical Faraday effect induced by pump pulses with a photon energy of $3.1 \mathrm{eV}$ in magnetic fields of opposite polarity. A field of $0.25 \mathrm{~T}$ is applied at an angle $\sim 20$ deg from the sample surface. The temperature is $10 \mathrm{~K}$. (b) Odd and even dynamics with respect to the applied magnetic field: $\alpha_{F}\left(H_{o}\right)=\alpha_{F}\left(H^{+}\right)-\alpha_{F}\left(H^{-}\right)$and $\alpha_{F}\left(H_{e}\right)=\alpha_{F}\left(H^{+}\right)+\alpha_{F}\left(H^{-}\right)$, respectively. (c) Transient magneto-optical Faraday effect induced by pump of opposite helicities and photon energies of $3.1 \mathrm{eV}$. (d) Odd $\alpha_{F}\left(\sigma_{o}\right)=\alpha_{F}\left(\sigma^{+}\right)-\alpha_{F}\left(\sigma^{-}\right)$and even $\alpha_{F}\left(\sigma_{o}\right)=\alpha_{F}\left(\sigma^{+}\right)+\alpha_{F}\left(\sigma^{-}\right)$ dynamics with respect to the pump helicity.

polarities of the applied magnetic field $\left(\mathrm{H}^{+}\right.$and $\left.\mathrm{H}^{-}\right)$. From Fig. 3(a) it can be seen that the oscillations are sensitive to the field polarity, being an odd function of the applied magnetic field. To emphasize this fact, we calculated the odd $\alpha_{F}\left(H_{o}\right)=\alpha_{F}\left(H^{+}\right)-\alpha_{F}\left(H^{-}\right)$and even parts of the measured dynamic signals $\alpha_{F}\left(H_{e}\right)=\alpha_{F}\left(H^{+}\right)+\alpha_{F}\left(H^{-}\right)$. From the graphs, shown in Fig. 3(b), it is seen that the oscillations are only seen in the odd part.

The dynamics of the laser-induced probe polarization rotation is shown in Fig. 3(c) for two pump helicities. Similarly, we calculated the helicity odd and helicity even parts of the measured dynamics: $\alpha_{F}\left(\sigma_{o}\right)=\alpha_{F}\left(\sigma^{+}\right)-\alpha_{F}\left(\sigma^{-}\right)$and $\alpha_{F}\left(\sigma_{o}\right)=\alpha_{F}\left(\sigma^{+}\right)+\alpha_{F}\left(\sigma^{-}\right)$. In Fig. 3(d) one can see that for the pump photon energy of $3.1 \mathrm{eV}$ the oscillations do not depend on the helicity of the polarization of the pump pulses.

The observed helicity-even but field-odd oscillations exhibit all the features of coherent spin precession triggered as a result of laser-induced heating [3,31]. In thermodynamic equilibrium the magnetization vector is aligned along the effective magnetic field, which is defined from the free energy as $\mu_{0} \vec{H}_{\text {eff }}=-\frac{\partial \Phi}{\partial \vec{M}}$, where $\Phi$ is the free energy of the magnet. Distinguishing the magnetization independent $\boldsymbol{\Phi}_{0}$ from magnetization dependent parts of the free energy one can write $\Phi=\Phi_{0}+2 \pi M_{z}^{2}-\mu_{0} \vec{M} \vec{H}_{a}-\mu_{0} \vec{M} \vec{H}_{\text {ext }}$, where $\vec{H}_{\text {ext }}$ is the external magnetic field, $\vec{H}_{a}$ is the magnetocrystalline anisotropy field aligned in the sample plane, and $\boldsymbol{M}_{\boldsymbol{z}}$ is the magnetization component normal to the sample plane. The photon energy of $3.1 \mathrm{eV}$ is much higher than the band gap of $\mathrm{CdCr}_{2} \mathrm{Se}_{4}$. This means that pump pulses excite electrons far into the conduction band. As a result of the strong absorption, the temperature of the electrons increases. The hot electrons interact with the lattice on a time scale of the electron-phonon 

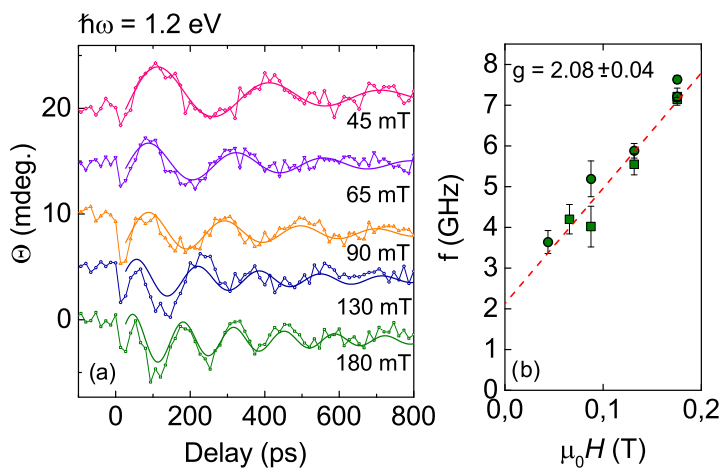

FIG. 4. (a) Transient magneto-optical Faraday rotation induced by pumping with a photon energy of $1.2 \mathrm{eV}$ at different magnetic fields, measured at $10 \mathrm{~K}$. The external magnetic field is applied in the plane of the sample. (b) The frequency of the oscillations observed in panel (a) as a function of the applied magnetic field. The frequencies were deduced with the help of FFT analysis. Circles and squares correspond to two different circular polarizations of the pump.

interaction resulting in a lattice heating and a change of the strength of the magnetocrystalline anisotropy $\vec{H}_{a}[32,33]$. On a timescale of spin-lattice coupling the temperature of the spin system also increases, resulting in a decrease of the magnetization $|\vec{M}|$. Moreover, due to the $s d$-exchange interaction in the conduction band and the $p d$-exchange interaction in the valence band, ultrafast laser-induced demagnetization can be caused by scattering of hot charge carriers on localized spins of $\mathrm{Cr}^{3+}$ ions. A decrease of $|\vec{M}|$ affects all corresponding magnetization dependent terms of the free energy. However, for a polycrystalline film, as the one discussed here, $\vec{H}_{a}$ can be neglected. Therefore, such a laser excitation changes the orientation of $\vec{H}_{\text {eff }}$, changing the equilibrium orientation for the magnetization. In response to this change, the magnetization starts precessing around the new equilibrium orientation, resulting in the observed oscillations.

\section{B. Pump photon energies below the band gap}

We also performed time-resolved measurements of the probe polarization rotation induced by circularly polarized pump photons with an energy below the band gap. Earlier it was reported that pumping the semiconductor with light can effectively induce polarization dependent domain-wall motion and a change of the net magnetization, with the maximum of efficiency at a photon energy of $1.2 \mathrm{eV}[27,34]$.

In our experiments we first suppressed possible manifestation of the polarization independent heating-induced mechanism of spin precession by applying an external magnetic field in the plane of the sample. If the pump photon energy is tuned to $1.2 \mathrm{eV}$, the observed dynamics clearly reveal oscillations [Fig. 4(a)]. As in the previous part, we studied the laser-induced dynamics as a function of the applied magnetic field. The FFT analysis demonstrates that the frequency of the oscillations is a linear function of the applied magnetic field with $g$ factor close to 2 [shown in Fig. 4(b)]. The dynamics of the polarization rotation measured for pump photon energies of $1.2 \mathrm{eV}$ at different temperatures is shown in Fig. 5(a). The frequency of the oscillations slightly softens close to the Curie
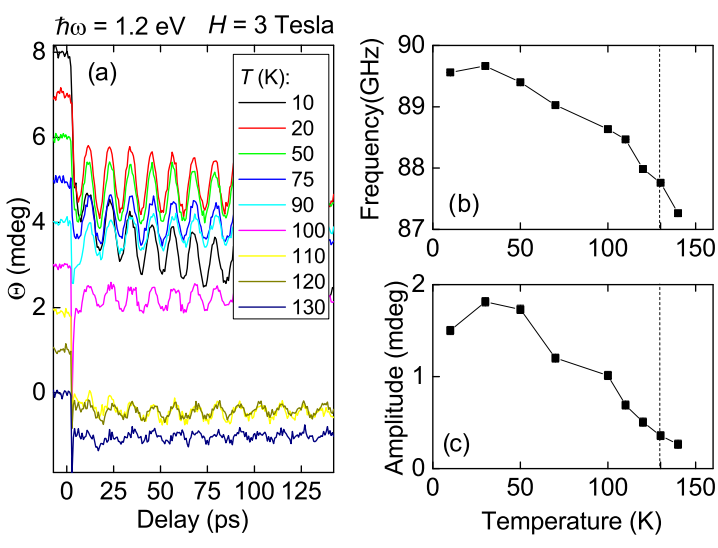

FIG. 5. (a) Transient magneto-optical Faraday effect induced by pump pulses with a photon energy of $1.2 \mathrm{eV}$ measured at different temperatures at $3 \mathrm{~T}$. The external magnetic field was applied in the plane of the sample. Both (b) and (c) show a decreasing frequency and amplitude of the oscillations, deduced from the FFT analysis.

point [see Fig. 5(b)], and the amplitude drops to zero above the critical temperature [Fig. 5(c)]. All these dependencies suggest that the observed effect can be assigned to the laserinduced homogeneous spin precession at the frequency of ferromagnetic resonance.

To reveal the mechanism of the laser-induced dynamics in this regime, we studied the dynamics under changing the polarity of the applied magnetic field as well as the helicity of the circularly polarized pump. In contrast to the measurements with the pump photon energy of $3.1 \mathrm{eV}$, for $1.2 \mathrm{eV}$ the polarization rotation is independent of the polarity of the applied magnetic field [see Fig. 6(a)]. Taking the difference
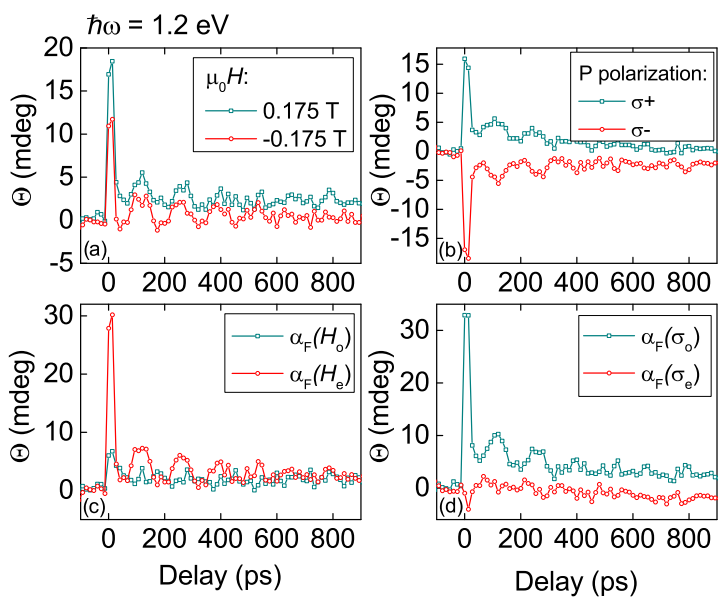

FIG. 6. (a) Transient magneto-optical Faraday effect induced by pump pulses with a photon energy of $1.2 \mathrm{eV}$ in magnetic fields of opposite polarities. The field of $0.175 \mathrm{~T}$ is applied in the plane of the sample surface. The temperature is $10 \mathrm{~K}$. (b) Odd and even dynamics with respect to the applied magnetic field: $\alpha_{F}\left(H_{o}\right)=\alpha_{F}\left(H^{+}\right)-$ $\alpha_{F}\left(H^{-}\right)$and $\alpha_{F}\left(H_{e}\right)=\alpha_{F}\left(H^{+}\right)+\alpha_{F}\left(H^{-}\right)$, respectively. (c) Transient magneto-optical Faraday effect induced by pump pulses of opposite helicities and photon energies of $1.2 \mathrm{eV}$. (d) Odd $\alpha_{F}\left(\sigma_{o}\right)=$ $\alpha_{F}\left(\sigma^{+}\right)-\alpha_{F}\left(\sigma^{-}\right)$and even $\alpha_{F}\left(\sigma_{o}\right)=\alpha_{F}\left(\sigma^{+}\right)+\alpha_{F}\left(\sigma^{-}\right)$dynamics with respect to the pump helicity. 


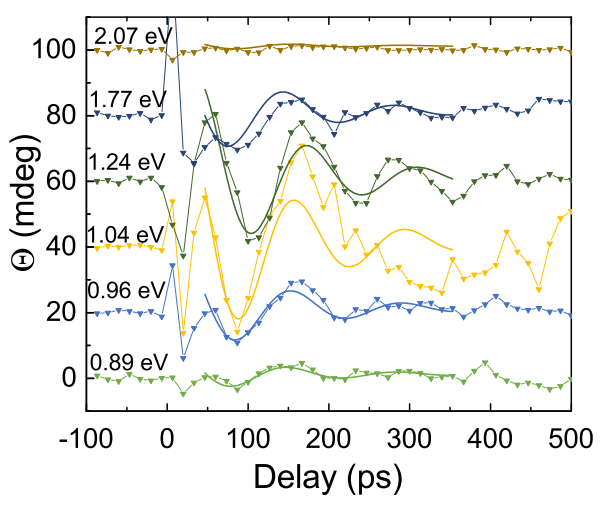

FIG. 7. Pump photon energy dependent transient magnetooptical Faraday effect. All curves are obtained by subtracting the single-exponential relaxation with $\tau=8$ ps. Probe photon energy is $1.55 \mathrm{eV}$. Pump polarization is $\sigma^{-}$. The field of $0.175 \mathrm{~T}$ is applied in the plane of the sample surface. The temperature is $10 \mathrm{~K}$.

between the transient rotation signals, measured in opposite magnetic fields, $\alpha_{F}\left(H_{o}\right)=\alpha_{F}\left(H^{+}\right)-\alpha_{F}\left(H^{-}\right)$, cancels the oscillations completely, suggesting that the mechanism is different from the one based on laser-induced heating. Moreover, our experiments with pump pulses of opposite circular polarization reveal that now the spin precession does depend on the helicity of the pump photons [Fig. 6(c)]. Changing the helicity of the pump pulses, one also changes the sign of the oscillations. In Fig. 6(d) we plot the difference between the outcomes of the experiments with the left- and right-handed circularly polarized pump beams. It is seen that the oscillations completely disappear in the even part of the signal, $\alpha_{F}\left(\sigma_{o}\right)=\alpha_{F}\left(\sigma^{+}\right)+\alpha_{F}\left(\sigma^{-}\right)$. Moreover, the fast noncoherent signal, observed as picosecond relaxation, also changes sign with the pump helicity.

The dependence on the polarity of the magnetic field and on the helicity of light suggests that the homogeneous spin precession at the frequency of ferromagnetic precession, induced by pump photons with an energy of $1.2 \mathrm{eV}$, cannot be excited via the heating mechanism, but must be explained by taking into account the electronic structure of the semiconductor.

To reveal the electronic transitions responsible for the helicity dependent laser-induced spin dynamics, we tuned the photon energy of the pump pulses around the energy of the band gap. In order to exclude heat-assisted polarization independent mechanisms of spin precession, we applied the magnetic field in the plane of the sample. Figure 7 shows the laser-induced transients of the magneto-optical signal. We can clearly observe oscillations in a wide region of pump photon energies from 0.89 to $1.77 \mathrm{eV}$, which completely disappear at $2.07 \mathrm{eV}$. It is seen that the phase and frequency of the spin oscillations also depend on the pump photon energy. However, with the available signal to noise ratio we cannot draw any quantitative conclusions about such dependencies.

We found that the amplitude has a maximum around $1.2 \mathrm{eV}$, which is very close to the band gap of the semiconductor [see Fig. 8(a)]. Regarding such a spectral dependence with a maximum corresponding to the maximum in the absorption spectrum [35], we suggest that the mechanism of excitation

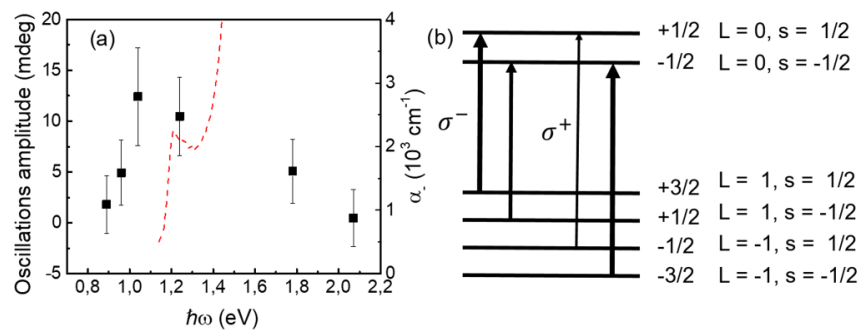

FIG. 8. (a) Amplitude of the spin oscillations at the frequency of ferromagnetic resonance induced by circularly polarized laser pulses. The amplitudes were deduced from the transient Faraday rotation traces by fitting the oscillations with a sine function. The measurements were performed at $10 \mathrm{~K}$ in an in-plane external magnetic field of $0.175 \mathrm{~T}$. Dashed line is the spectral dependence of the optical absorption coefficient for $\sigma^{-}$polarization, adopted from [27]. (b) Schematic of the $\mathrm{CdCr}_{2} \mathrm{Se}_{4}$ band structure below the magnetic ordering temperature. Arrows show transitions observed in the experiment.

of spin precession can be explained by optical spin transfer torque, similarly to Ref. [19].

According to Ref. [36] this resonance corresponds to the transition from the top of the valence band to the localized $d$ states of $\mathrm{Cr}^{3+}$ ions. Alternatively, the energy of the observed maximum can correspond to the transition from the top of the valence band, formed by $4 p$ electrons of $\mathrm{Se}^{4+}$ ions, to the bottom of the conduction band, formed by $4 s$ electrons of $\mathrm{Cr}^{3+}$ ions. In this case, the mechanism of excitation of the polarization dependent spin precession can rely on spin-orbit interaction and $p d$-exchange interaction experienced by charge carriers in the valence band. In particular, a strong spin-orbit interaction experienced by the carriers in the valence band is responsible for the coupling of the spin of the photogenerated carriers and the polarization of light. Due to strong $s p-d$ exchange interactions the spins of the photocarriers appear to be coupled to the network of ordered spins of $\mathrm{Cr}^{3+}$ ions.

Figure 8(b) demonstrates a scheme of the electronic transitions triggered in the semiconductor by circularly polarized light. The scheme takes into account strong spin-orbit interaction in the valence band $[37,38]$ as well as spin splitting of the valence and the conduction bands [36] induced by the $p d$ and the $s d$-exchange interactions, respectively. Similar to the case of optical orientation in direct band-gap semiconductors, the transitions from the sub-band of light holes $(L=-1, s=$ $+1 / 2 ; L=+1, s=-1 / 2)$ are less intense than those from the heavy-hole sub-band $(L=+1, s=+1 / 2 ; L=-1, s=$ $-1 / 2$ ). Therefore, pumping with circularly polarized light effectively generates a nonequilibrium spin polarization in the valence band, the orientation of which is defined by the helicity of light. The nonequilibrium spin polarization is exchange coupled to the ordered $\mathrm{Cr}$ spins and acts as an effective magnetic field, through the optical spin transfer torque mechanism, similarly to the case discussed in GaMnAs [19]. Also pumping the electrons to the localized $d$ states $\mathrm{Cr}^{3+}$ ions and creating a nonequilibrium spin polarization in the excited states will act as an effective magnetic field. Analyzing the measured time traces one can clearly distinguish a very sharp helicity dependent peak with a relaxation time $\tau_{1}=8 \mathrm{ps}$ 
(see Fig. 6). This relaxation time can be assigned to the spin lifetime of the photocarriers [39,40].

Notably, there is one more relaxation process observed in the transient rotation signal. The relaxation time is $\tau_{2}=$ $1 \mathrm{~ns}$ [see Fig. 6(d)]. In analogy with [40], where a similar time constant was associated with the lifetime of carriers on localized levels, we suggest that $\tau_{2}$ corresponds to the lifetime of the photoexcited electrons on $\mathrm{Cr}^{3+}$ levels.

\section{CONCLUSIONS}

To summarize, we report about two mechanisms of photoinduced spin dynamics in the magnetic semiconductor $\mathrm{CdCr}_{2} \mathrm{Se}_{4}$. Photons with an energy higher than the band gap heat up the electrons, effectively change the equilibrium orientation of the magnetization, and thus displacively excite homogeneous spin precession at the frequency of ferromagnetic resonance. The second mechanism implies that circularly polarized light can act on spins as an effective magnetic field. In $\mathrm{CdCr}_{2} \mathrm{Se}_{4}$ this effect is strongly wavelength dependent, and has a maximum at photon energies of $1.04 \mathrm{eV}$. This energy is close to the electronic transition from the valence band to the bottom of the conduction band. Due to spin-orbit interaction light excites carriers with a given spin and due to $s p$ - $d$ exchange interaction this excitation is transferred to the spins of the $\mathrm{Cr}^{3+}$ ions. Although the nonequilibrium spin polarization relaxes with a decay time of $8 \mathrm{ps}$, upon this relaxation the nonequilibrium spin polarization manages to trigger oscillations of $\mathrm{Cr}$ spins due to spin transfer torque.

\section{ACKNOWLEDGMENTS}

We would like to thank Prof. N. Bebenin for fruitful discussions. This work was supported by de Nederlandse Organisatie voor Wetenschappelijk Onderzoek (NWO), the European Union's Seventh Framework Program (FP7/20072013)/European Research Council Grants No. 339813 (Exchange) and No. 257280 (Femtomagnetism), the state assignment of the Federal Agency for Scientific Organizations of the Russian Federation (Grant No. AAAA-A18118020290104-2 "Spin"), and the program "Leading Scientist" of the Russian Ministry of Education and Science (Grants No. 14.Z50.31.0025 and No. 14.z50.31.0034). We would like to thank Tonnie Toonen and Sergey Semin for technical support.
[1] E. Beaurepaire, J.-C. Merle, A. Daunois, and J.-Y. Bigot, Ultrafast Spin Dynamics in Ferromagnetic Nickel, Phys. Rev. Lett. 76, 4250 (1996).

[2] A. V. Kimel, A. Kirilyuk, P. A. Usachev, R. V. Pisarev, A. M. Balbashov, and Th. Rasing, Ultrafast non-thermal control of magnetization by instantaneous photomagnetic pulses, Nature (London) 435, 655 (2005).

[3] F. Hansteen, A. V. Kimel, A. Kirilyuk, and Th. Rasing, Femtosecond Photomagnetic Switching of Spins in Ferrimagnetic Garnet Films, Phys. Rev. Lett. 95, 047402 (2005).

[4] A. V. Kimel, B. A. Ivanov, R. V. Pisarev, P. A. Usachev, A. Kirilyuk, and Th. Rasing, Inertia-driven spin switching in antiferromagnets, Nat. Phys. 5, 727 (2009).

[5] A. Stupakiewicz, K. Szerenos, D. Afanasiev, A. Kirilyuk, and A. V. Kimel, Ultrafast nonthermal photo-magnetic recording in a transparent medium, Nat. Publ. Gr. 542, 71 (2017).

[6] B. Koopmans, G. Malinowski, F. Dalla Longa, D. Steiauf, M. Fahnle, T. Roth, M. Conchetti, and M. Aeschlimann, Explaining the paradoxical diversity of ultrafast laser-induced demagnetization, Nat. Mater. 9, 259 (2010).

[7] C. Boeglin, E. Beaurepaire, V. Halte, V. Lopez-Flores, C. Stamm, N. Pontius, H. A. Durr, and J.-Y. Bigot, Distinguishing the ultrafast dynamics of spin and orbital moments in solids, Nature (London) 465, 458 (2010).

[8] C. D. Stanciu, F. Hansteen, A. V. Kimel, A. Kirilyuk, A. Tsukamoto, A. Itoh, and Th. Rasing, All-Optical Magnetic Recording with Circularly Polarized Light, Phys. Rev. Lett. 99, 047601 (2007).

[9] A. Kirilyuk, A. V. Kimel, and Th. Rasing, Ultrafast optical manipulation of magnetic order, Rev. Mod. Phys. 82, 2731 (2010)

[10] R. R. Subkhangulov, A. B. Henriques, P. H. O. Rappl, E. Abramof, Th. Rasing, and A. V. Kimel, All-optical manipula- tion and probing of the $d-f$ exchange interaction in EuTe, Sci. Rep. 4, 4368 (2014).

[11] R. R. Subkhangulov, H. Munekata, Th. Rasing, and A. V. Kimel, Laser-induced spin dynamics in ferromagnetic (In,Mn)As at magnetic fields up to 7 T, Phys. Rev. B 89, 060402(R) (2014).

[12] J. Wang, C. Sun, J. Kono, A. Oiwa, H. Munekata, L. Cywinski, and L. J. Sham, Ultrafast Quenching of Ferromagnetism in InMnAs Induced by Intense Laser Irradiation, Phys. Rev. Lett. 95, 167401 (2005).

[13] J. Wang, G. A. Khodaparast, J. Kono, A. Oiwa, and H. Munekata, Ultrafast magneto-optics in ferromagnetic III-V semiconductors, J. Phys.: Condens. Matter 18, R501 (2006).

[14] K. Sekiguchi, K. Yamada, S. M. Seo, K. J. Lee, D. Chiba, K. Kobayashi, and T. Ono, Nonreciprocal emission of spinwave packet in FeNi film, Cit. Appl. Phys. Lett 97, 022508 (2010).

[15] T. Makino, F. Liu, T. Yamasaki, Y. Kozuka, K. Ueno, A. Tsukazaki, T. Fukumura, Y. Kong, and M. Kawasaki, Ultrafast optical control of magnetization in EuO thin films, Phys. Rev. B 86, 064403 (2012).

[16] F. Liu, T. Makino, T. Yamasaki, K. Ueno, A. Tsukazaki, T. Fukumura, Y. Kong, and M. Kawasaki, Ultrafast TimeResolved Faraday Rotation in EuO Thin Films, Phys. Rev. Lett. 108, 257401 (2012).

[17] M. Matsubara, A. Schroer, A. Schmehl, A. Melville, C. Becher, M. Trujillo-Martinez, D. G. Schlom, J. Mannhart, J. Kroha, and M. Fiebig, Ultrafast optical tuning of ferromagnetism via the carrier density, Nat. Commun. 6, 6724 (2015).

[18] N. Tesařová, P. Němec, E. Rozkotová, J. Zemen, T. Janda, D. Butkovičová, F. Trojánek, K. Olejník, V. Novák, P. Malý, and T. Jungwirth, Experimental observation of the optical spin-orbit torque, Nat. Photon. 7, 492 (2013). 
[19] P. R Němec, E. Rozkotová1, N. Tesarová, F. Trojánek, E. De Ranieri, K. Olejník, J. Zemen, V. Novák, M. Cukr, P. Malý, and T. Jungwirth, Experimental observation of the optical spin transfer torque, Nat. Phys. 8, 411 (2012).

[20] G. Harbeke and H. W. Lehmann, Optical transitions and band structure model for cadmium chromium chalcogenides, Solid State Commun. 8, 1281 (1970).

[21] D. M. Eagles, Optical absorption due to transitions between bands and local levels in $\mathrm{CdCr}_{2} \mathrm{Se}_{4}$, J. Phys. Chem. Solids 39, 1243 (1978).

[22] V. T. Moshnyaga, V. B. Anzin, V. G. Veselago, M. Golant, and M. A. Chernikov, Surface and volume absorption of light in the magnetic semiconductor $\mathrm{CdCr}_{2} \mathrm{Se}_{4}$, J. Exp. Theor. Phys. 58, 562 (1983).

[23] A. A. Samokhvalov, V. V. Osipov, N. I. Solin, A. F. Gunichev, I. A. Korenblit, and A. P. Galdikas, Electron-magnon interaction in magnetic semiconductors, J. Magn. Magn. Mater. 46, 191 (1984).

[24] T. Ogasawara, K. Ohgushi, Y. Tomioka, K. S. Takahashi, H. Okamoto, M. Kawasaki, and Y. Tokura, General Features of Photoinduced Spin Dynamics in Ferromagnetic and Ferrimagnetic Compounds, Phys. Rev. Lett. 94, 087202 (2005).

[25] V. G. Veselago, A. A. Minakov, and S. G. Rudov, Direct observation of photoinduced change in magnetocrystalline anisotropy in $\mathrm{CdCr}_{2} \mathrm{Se}_{4}: \mathrm{Ga}$, J. Exp. Theor. Phys. 60, 360 (1984).

[26] G. M. Genkin, Y. N. Nozdrin, I. D. Tokman, and V. N. Shastin, Direct observation of photomagnetization of the ferromagnet $\mathrm{CdCr}_{2} \mathrm{Se}_{4}$ by circularly polarizad light, JETP Lett. 35, 199 (1982).

[27] L. L. Golik and Z. E. Kunkova, Non-linearities in the Faraday rotation and absorption due to excitons in the ferromagnetic semiconductor $\mathrm{CdCr}_{2} \mathrm{Se}_{4}$, Phys. Status Solidi 219, 411 (2000).

[28] H. W. Lehmann and J. M. Harbeke, Semiconducting and optical properties of ferromagnetic $\mathrm{CdCr}_{2} \mathrm{~S}_{4}$ and $\mathrm{CdCr}_{2} \mathrm{Se}_{4}$, J. Appl. Phys. 38, 946 (1967).

[29] V. N. Berzhansky, N. A. Drokin, V. I. Ivanov, V. P. Kononov, I. S. Edelman, S. A. Havrichkov, V. K. Chernov, A. G. Shishkov, and A. M. Pirogova, Synthesis and physical properties of chalcogenide chromium spinel films, Thin Solid Films 190, 199 (1990).
[30] A. V. Kimel, G. V. Astakhov, A. Kirilyuk, G. M. Schott, G. Karczewski, W. Ossau, G. Schmidt, L. W. Molenkamp, and Th. Rasing, Observation of Giant Magnetic Linear Dichroism in (Ga,Mn)As, Phys. Rev. Lett. 94, 227203 (2005).

[31] C. D. Stanciu, A. Tsukamoto, A. V. Kimel, F. Hansteen, A. Kirilyuk, A. Itoh, and Th. Rasing, Subpicosecond Magnetization Reversal Across Ferrimagnetic Compensation Points, Phys. Rev. Lett. 99, 217204 (2007).

[32] L. A. Shelukhin, V. V. Pavlov, P. A. Usachev, Shamray P. Yu., R. V. Pisarev, and A. M. Kalashnikova, Ultrafast laser-induced changes of the magnetic anisotropy in a low-symmetry iron garnet film, Phys. Rev. B 97, 014422 (2018).

[33] J. Y. Bigot, M. Vomir, L. H. F. Andrade, and E. Beaurepaire, Ultrafast magnetization dynamics in ferromagnetic cobalt: The role of the anisotropy, Chem. Phys. 318, 137 (2005).

[34] V. G. Veselago, G. I. Vinogradova, A. A. Garmonov, S. G. Rudov, É. G. Zhukov, I. Kurbanklychev, and V. A. Levshin, Photoinduced increase in the magnetization in the region of a phase transition in the ferromagnetic semiconductors $\mathrm{CdCr}_{2} \mathrm{Se}_{4}$ and $\mathrm{HgCr}_{2} \mathrm{Se}_{4}$, J. Exp. Theor. Phys. 70, 311 (1990).

[35] S. G. Stoyanov, M. N. Iliev, and S. P Stoyanova, Spectral dependence of the photoconductance in $\mathrm{CdCr}_{2} \mathrm{Se}_{4}$ : band and donor level splitting due to ferromagnetic ordering, Solid State Commun. 18, 1389 (1976).

[36] N. Sanford, R. W. Davies, A. Lempicki, W. J. Miniscalco, and S. J. Nettel, Influence of Polarized Optical Pumping on the Ferromagnetism of $\mathrm{CdCr}_{2} \mathrm{Se}_{4}$, Phys. Rev. Lett. 50, 1803 (1983).

[37] V. A. Gavrichkov and S. G. Ovchinnikov, Multielectron energy structure and physical properties of the ferromagnetic semiconductor $\mathrm{CdCr}_{2} \mathrm{Se}_{4}$, J. Exp. Theor. Phys. 63, 744 (1986).

[38] M. I. Auslender and N. G. Bebenin, On the band structure and anisotropy of transport properties of ferromagnetic semiconductors $\mathrm{CdCr}_{2} \mathrm{Se}_{4}$ and $\mathrm{HgCr}_{2} \mathrm{Se}_{4}$, Solid State Commun. 69, 761 (1989).

[39] Y. Takagi, S. Adachi, S. Takeyama, A. Tackeuchi, S. Muto, and J. J. Dubowski, Ultrafast exciton spin relaxation in GaAs/AlGaAs and CdMnTe multiple quantum wells, J. Lumin. 58, 202 (1994).

[40] S. Bar-Ad and I. Bar-Joseph, Exciton Spin Dynamics in GaAs Heterostructures, Phys. Rev. Lett. 68, 349 (1992). 